\title{
Analysis of surface plasmon modes and band structures for plasmonic crystals in one and two dimensions
}

\author{
Ruey-Lin Chern, ${ }^{1, *}$ Chien C. Chang, ${ }^{2,1, \dagger}$ and C. Chung Chang ${ }^{2}$ \\ ${ }^{1}$ Institute of Applied Mechanics, National Taiwan University, Taipei 106, Taiwan, Republic of China \\ ${ }^{2}$ Division of Mechanics, Research Center for Applied Sciences, Academia Sinica, Taipei 115, Taiwan, Republic of China
}

(Received 25 October 2005; published 7 March 2006)

\begin{abstract}
In this study, we employ the interfacial operator approach to compute surface plasmon modes as well as band structures (including longitudinal modes) for plasmonic crystals in one and two dimensions. In particular, we consider the free-electron model for the metal. It is shown that the localized feature of surface plasmon modes can be resolved near the interface by introducing interfacial variables. For a one-dimensional array of metal, convergence of two branches of surface plasmon modes is studied by varying the filling fraction of the metal. For two-dimensional metallic structures, band flattening, band broadening, and plasmonic band gaps are observed and discussed. The highly degenerate nature and infinite number of surface plasmon modes can be explained by employing the Rayleigh quotient for the TE modes. The cutoff behavior in the TM modes is made clear by considering the energy density of the electromagnetic fields. The transverse electric fields, surface charges, and polarization currents are visualized to help understand various properties of surface plasmon modes. Moreover, the effect of plasma frequency and the transition from dispersive metals to perfect conductors are also explored. Finally, the contribution of Drude damping is considered by perturbation analysis.
\end{abstract}

DOI: 10.1103/PhysRevE.73.036605

PACS number(s): 42.70.Qs, 78.20.Bh, 02.70.Bf

\section{INTRODUCTION}

Periodic structures with dielectric materials have been extensively studied since 1987 [1,2]. Their optical properties mainly depend on the dielectric constant of the material, the geometry of the structure, and the direction of the polarization. If the structure contains metallic components, then the motion of the electrons will drastically alter the optical properties. In general, there are three levels to model the metal property. The first one is to assume metals to be perfect conductors. The second one is to model metals as dispersive materials with the simple free-electron form of the dielectric function. The third one is to model metals as dissipative materials with the Drude type of dielectric function. The most significant feature of periodic metallic structures is the existence of surface plasmon modes. And for this reason, periodic structures made of the latter two types of metals are also named plasmonic crystals $[3,4]$. Surface plasmon modes are highly localized around the interface of the metal and the surrounding dielectric. Also, intensive gathering of the surface plasmon modes around the surface plasma frequency $\omega_{s p}$ gives rise to high density of states and field enhancements. These features of surface plasmons may find various applications in optical data storage, miniaturized photonic circuits, surface-enhanced Raman scattering, biosensing, light generation, and solar cells [5-10].

It has been a difficult issue to compute surface plasmon modes and band structures for frequency-dependent materials, in particular for the TE modes. The free-electron model, though simple, enables us to investigate some very important optical properties of dispersive metallic structures. On the

\footnotetext{
*Electronic address: chern@iam.ntu.edu.tw

${ }^{\dagger}$ Electronic address: mechang@gate.sinica.edu.tw
}

one hand, the highly localized nature of surface plasmon polaritons requires very-fine-resolution schemes. On the other hand, the eigensystem for frequency-dependent materials no longer has a standard format that can be solved by standard eigenvalue solvers. In fact, it is referred to as one type of nonlinear eigenvalue problem [11], which is nonlinear in eigenfrequency. Besides, the change of type of the eigensystem across the interface of the metal and the dielectric material (with the dielectric constant positive in the dielectric and negative in the metal) makes the problem nondefinite, which adds further difficulty to compute the band structures. Nevertheless, a few methods have been applied for this frequency-dependent nonlinear eigenvalue problem. In the most commonly used plane-wave expansion method, the field is expanded by a series of Fourier components. The wave equation is then converted to a quadratic eigensystem [12]. In the finite-difference time-domain method, an oscillating dipole is introduced in the structure to excite the surface plasmon modes. The eigenfrequencies are obtained by Fourier transforming the time series of the resultant fields [13]. In the multiple-multipole method, the field is expanded as a linear superposition of analytical functions based on different expansion centers. A carefully designed cost function is used to test for the eigenfrequencies [14].

In one previous paper [15], we studied the effect of metallic components modeled as perfect conductors as well as dispersive metals for TM modes. Recently, we further proposed an interfacial operator approach to compute band structures for dispersive metals with the free-electron model [16]. In this study, we employ the interfacial operator approach to compute band structures for plasmonic crystals in one and two dimensions as shown in Fig. 1. For the layered structure [Fig. 1(a)], it will be shown that the frequencies of two surface plasmon modes converge to the surface plasma frequency $\omega_{s p}$ at large off-line wave numbers. Mode splitting 


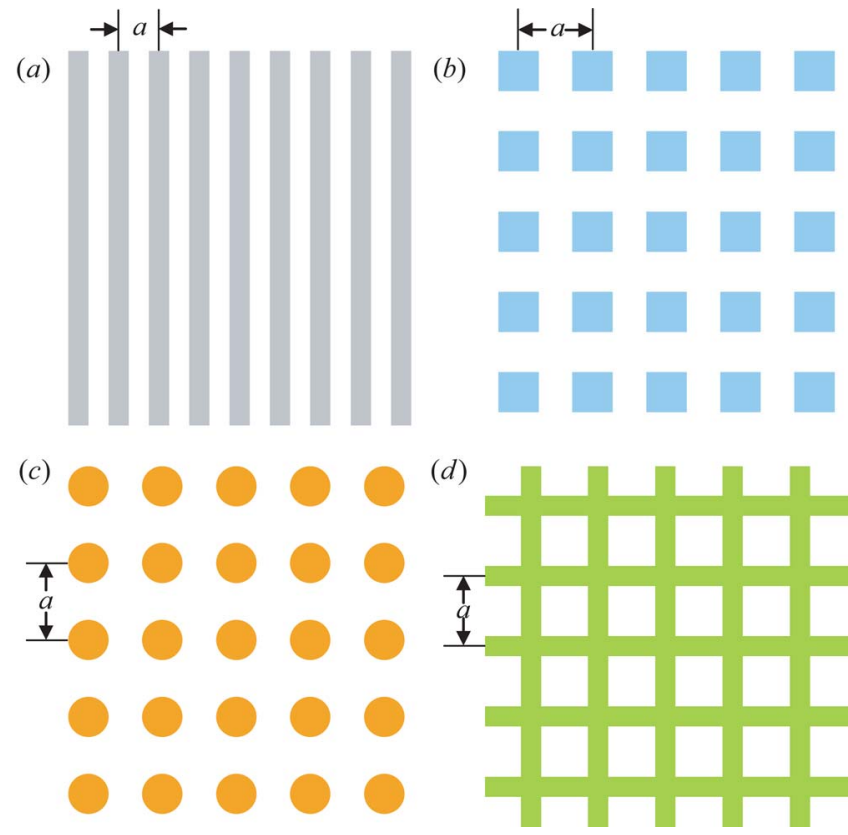

FIG. 1. (Color online) Plasmonic crystals made of dispersive metals. (a) One-dimensional layered structure, (b) two-dimensional (2D) array of square cylinders, (c) 2D array of circular cylinders, and (d) 2D array of grid cylinders.

and the convergence rate are related to the interaction of the modes from both sides of the metal as well as the dielectric. For two-dimensional structures [Figs. 1(b)-1(d)], very sharp feature of surface plasmon modes can be resolved in detail. In particular, surface plasmon polariton (SPP) modes and localized surface plasmon (LSP) modes are identified regarding their extending characteristics along the interface. The transverse electric fields, surface charges, and polarization currents for a typical surface plasmon mode are illustrated to study the properties of surface plasmon modes, as well as the active response of dispersive metals. For TE modes, we observed the phenomenon of a band flattening, the origin of which is explained through the help of the Rayleigh quotient for the eigensystem. In addition to band flattening, we also observed band broadening, which is more obvious for thin structures, due to lifting of the degeneracy by effective interaction of the modes. For moderately thick structure, a balance of band flattening and broadening may result in a possible plasmonic band gap around $\omega_{s p}$.

Another aspect of metallic structures is the cutoff behavior in the TM modes. In general, a larger fraction of metallic components may produce a higher cutoff frequency. This can be made clear by considering the energy density of the electromagnetic field for dispersive materials. For very high fractions of the metal, all the TM bands may lie above $\omega_{p}$ and coincide with the TE bands there, while the other TE bands below $\omega_{p}$ reduce to a few flatbands. Appearance of the cutoff frequency also implies that no static modes $(\omega=0)$ are allowed for any wave vectors in the TM bands. However, static modes do appear in the TE bands. The reason is explained on a unified basis by examining different types of boundary conditions for the TM and TE modes, respectively. It is found that a full or partial band of static modes with zero frequency for TE modes may exist if the metallic components are connected. The same phenomenon is also observed for the one-dimensional layered structure. As a result, large full band gaps between the first few frequency bands are opened. For isolated metallic structures, however, there is no similar behavior. In the present study, we also investigate the effect of the plasma frequency $\omega_{p}$ on surface plasmon modes and band structures. First of all, $\omega_{p}$ is a measure of how dispersive metals approach being perfect conductors. As $\omega_{p}$ is increased, both the TM and TE bands move to higher frequencies, for there is more energy contained in the metal. In particular, static modes at the point $\Gamma$ are illustrated to explore the evanescent fields and related skin depths in the metal. In the limiting case where $\omega_{p}$ approaches infinity, dispersive metals behave like perfect conductors and the surface plasma frequency $\omega_{s p}$ goes to infinity as well, and therefore no surface plasmon modes exist at finite frequencies. Moreover, longitudinal modes with eigenfrequency equal to $\omega_{p}$ can also be obtained with the present approach. These modes correspond to coherent oscillations at the bulk plasma frequency and also to zero of the dielectric function. Finally, we consider the effect of dissipation in terms of the Drude damping by developing the first-order perturbation analysis to correct the eigenfrequencies.

\section{BASIC EQUATIONS}

Based on the Maxwell theory, the wave equations for linear, isotropic, and nonmagnetic materials in two dimensions are given by

$$
\begin{array}{r}
-\frac{1}{\varepsilon} \nabla^{2} E=\left(\frac{\omega}{c}\right)^{2} E, \\
-\nabla \cdot\left(\frac{1}{\varepsilon} \nabla H\right)=\left(\frac{\omega}{c}\right)^{2} H
\end{array}
$$

for the TM and TE modes, respectively, assuming that the fields vary harmonically in time with frequency $\omega$ as $e^{-i \omega t}$. The eigenmodes are solutions of the wave equations bound by some constraints. In this study, the periodicity of the structure serves as a constraint to the wave equations. For periodic structures, it is sufficient to solve the problem on one unit cell, along with the Bloch conditions

$$
\begin{aligned}
& E\left(\mathbf{r}+\mathbf{a}_{i}\right)=e^{\mathbf{i k} \cdot \mathbf{a}_{i}} E(\mathbf{r}), \\
& H\left(\mathbf{r}+\mathbf{a}_{i}\right)=e^{\mathbf{i k} \cdot \mathbf{a}_{i}} H(\mathbf{r}),
\end{aligned}
$$

where $\mathbf{k}$ is the wave vector and $\mathbf{a}_{i}(i=1,2)$ are the lattice translation vectors. [17]

In the present study, we consider the free-electron model

$$
\varepsilon(\omega)=1-\frac{\omega_{p}^{2}}{\omega^{2}}
$$

for the metal, where $\omega_{p}$ is the bulk plasma frequency. It is noted that in the free-electron model, the dielectric function of the metal is real and the conductivity is purely imaginary. 
That means the conductivity is $90^{\circ}$ out of phase with the field and there is no loss with this model. However, in the optical range of frequencies, dissipation due to electron collisions cannot be ignored. For this purpose, the Drude model [18]

$$
\varepsilon(\omega)=1-\frac{\omega_{p}^{2}}{\omega^{2}+i \gamma \omega}
$$

is employed to consider the contribution of damping. The appearance of the collision frequency $\gamma$ makes the dielectric function complex, and the allowed frequencies of the electromagnetic field become complex as well, resulting in a decay factor of the field in the time domain.

For the dielectric function (5), the eigensystems (1) and (2) can be written in the format

$$
\mathcal{L}(\Lambda) \phi=\Lambda \phi,
$$

where $\Lambda=\omega^{2} / c^{2}$ is the eigenvalue and $\phi$ is the eigenfunction, which can be either the $E$ or $H$ field. Note that the eigenvalue $\Lambda$ itself appears in the solution operator $\mathcal{L}$. If we discretize Eq. (7) in a straightforward manner-for example, by a finite-difference scheme-we will obtain a nonlinear discrete eigensystem

$$
\mathbf{B}(\Lambda) \mathbf{y}=\Lambda \mathbf{y},
$$

where $\mathbf{B}$ is the system matrix and $\mathbf{y}$ is the eigenvector. This is one type of nonlinear eigenvalue problem-that is, nonlinear in eigenfrequency. Equation (8) does not have a standard format that can be solved by standard eigenvalue solvers such as QR or inverse iteration [19]. However, for the TM modes, Eq. (1) can be rearranged to yield

$$
\left(-\nabla^{2}+\Lambda_{p}\right) E=\Lambda E,
$$

which is now a standard linear eigensystem, where $\Lambda_{p}$ $=\omega_{p}^{2} / c^{2}$. Equation (9) has been used to study the effect of metallic components embedded in the dielectric structure [15]. Nevertheless, this cannot be done for the TE modes because the dielectric function lies inside the operator. Surface plasmon modes may appear at the interface between the metal and dielectric for the TE modes. The highly localized nature of surface plasmon modes requires very fine resolution near the interface, across which the dielectric function changes its sign. This is equivalent to the change of type of Eq. (2) across the interface, adding further difficulty to obtain the solutions numerically.

\section{INTERFACIAL OPERATOR APPROACH}

The present authors have developed the interfacial operator approach [16] to solve the band structures as well as surface plasmon modes in one and two dimensions. This is a direct approach to solve the eigensystem (2) within the finite-difference framework. The basic idea is first to deal with the eigensystem (2) in the regions of the dielectric and metal separately so that the dielectric function can be moved out of the Laplace operator to yield

$$
-\frac{1}{\varepsilon_{d}} \nabla^{2} H=\Lambda H
$$

$$
\left(-\nabla^{2}+\Lambda_{p}\right) H=\Lambda H
$$

for points in the strict insides of the dielectric and metal, respectively, where $\varepsilon_{d}$ is the dielectric constant of the dielectric medium. The two equations (10) and (11) are then joined together with the interface condition

$$
\left[\frac{1}{\varepsilon} \frac{\partial H}{\partial n}\right]_{S}=0
$$

where $\partial / \partial n$ denotes the derivative in the surface normal direction and []$_{S}=0$ denotes the jump across the interface $S$. The interface condition (12) is obtained by integrating both sides of the eigensystem (2) over a thin box located on the interface and taking the limit as the box height goes to zero. The crucial step of the interfacial operator is to introduce an interfacial variable $R$ into the discretized form of the interfacial condition (12) and reformulate the eigensystem in terms of the $H$ field in the strict insides of the dielectric and metal, along with this new variable $R$. Based on the finite-difference formulation, the difference equation (12) is rearranged so that the eigenvalue $\Lambda$ only appears on the right-hand side to fulfill the standard format of the eigensystem. Next, the $H$ field at the interface that appears in the difference equation in the strict insides of the dielectric and metal is replaced by $R$. Combining the discretization of Eqs. (10) and (11), together with the reformulation of Eq. (12), we obtain a resultant eigensystem

$$
\mathbf{A x}=\Lambda \mathbf{x} .
$$

The system matrix $\mathbf{A}$ differs from $\mathbf{B}$ in two ways: (a) the eigenvalue $\Lambda$ does not appear in the operator $\mathbf{A}$, and (b) the $H$ field at the interface, being replaced by the interfacial variable $R$, does not appear in $\mathbf{x}$. However, the eigensystems (8) and (13) are equivalent to each other in the discrete sense that the eigenvalue $\Lambda$ is the same for both eigensystems and the eigenvector $\mathbf{x}$ can be converted to the original eigenvector $\mathbf{y}$ and vice versa. Nevertheless, the eigensystem (13) has the great advantage of being a standard format that can be solved by standard eigenvalue solvers. The detailed formulation of the interfacial operator approach is referred to Ref. [16].

Having obtained the solutions of Eqs. (1) and (2) with the free-electron model (5), the contribution of Drude damping can be considered by using the perturbation technique, applied with the interfacial operator approach. Ordinarily, the collision frequency $\gamma$ is small compared to the frequency $\omega$ such that $\gamma \ll \omega$. This suggests to us to regard the solution with damping based on the Drude model (6) as a perturbation to the undamped solution based on the free-electron model. Here, we refer to the standard perturbation theory [20] to obtain the first-order correction of the eigenvalue. First, the operator of the eigensystem $\mathcal{L} \phi=\Lambda \phi$ is split into two parts as

$$
\mathcal{L}=\mathcal{L}_{0}+\mathcal{L}_{1},
$$

where $\mathcal{L}_{0}$ is the operator of the undamped eigensystem. Assume the eigenvalue $\Lambda$ and eigenfunction $\phi$ be expanded as

$$
\Lambda=\Lambda_{0}+\Lambda_{1}+\cdots,
$$




$$
\phi=\phi_{0}+\phi_{1}+\cdots,
$$

where $\Lambda_{0}$ and $\phi_{0}$ are the eigenvalue and eigenfunction, respectively, of the operator $\mathcal{L}_{0}$. The correction of the eigenfrequency $\omega_{1}$ is then given as

$$
\omega_{1}=c\left(\sqrt{\Lambda_{0}+\Lambda_{1}}-\sqrt{\Lambda_{0}}\right) .
$$

Since the perturbed eigenvalues are in general complex, so are the perturbed eigenfrequencies. The imaginary part of the eigenfrequency results in a decay factor of the electromagnetic field in the time domain, which tells the effect of Drude damping.

\section{RESULTS AND DISCUSSIONS}

Surface plasmons are waves that propagate along the surface of a conductor, due to collective oscillation of electric charges coupled with the electromagnetic fields [21]. Because of the hybrid or mixing nature, surface plasmons are also named surface plasmon polaritons [22]. Under suitable conditions, surface plasmons may appear at the interface across which the dielectric constant changes sign. Ordinarily, it is the interface between a dielectric and a metal at some frequency range. It is noted that the electromagnetic field is transverse in nature, for the electric field satisfies the divergence free in a homogeneous medium $(\boldsymbol{\nabla} \cdot \mathbf{E}=0)$. On the other hand, the plasma or electron oscillation is longitudinal in nature, which corresponds to zero of the dielectric function $(\varepsilon=0)$. Basically, the two motions are not coupled with each other. But the presence of the interface gives the field a longitudinal component due to a discontinuity of the dielectric function across the interface $(\boldsymbol{\nabla} \cdot \mathbf{E}=-\mathbf{E} \cdot \boldsymbol{\nabla} \ln \varepsilon \neq 0)[23]$. Therefore, the motions of the electrons and electromagnetic fields could be coupled at the interface. However, because the fields inside the conductor have no longitudinal components to couple with the plasma oscillations, they rapidly decay away from the interface. Therefore, surface plasmons are highly localized at the interface and evanescent otherwise. It is also noted that only in the TE modes can surface plasmon modes be sustained. This is because in the TM modes the $E$ field is always tangential to the interface and continuous over there $\left([E]_{S}=\sigma=0\right)$, and therefore no surface charges exist to support surface plasmons. On the other hand, in the TE modes the $E$ field has a component across the interface, which allows the surface charges to exist $\left([E]_{S}\right.$ $=\sigma \neq 0)$.

Due to the binding of electrons, surface plasmons have larger momenta or, equivalently, larger wave numbers than the light wave at the same frequency. Therefore, in order to excite surface plasmons by light, some coupling mechanisms have to be introduced to cover the momentum deficit [23]. In the prism coupling technique, attenuated total reflection is applied on a thin metal film to provide a larger wave number by reducing the wave speed. In the grating coupling technique, the wave number is increased by an amount equal to the Fourier component of the periodicity of the grating. Another way to excite the surface plasmons is using the lattice coupling to supply additional dispersive modes. In this case, multiple scattering of the fields within the periodic structure is responsible for providing extra momenta.

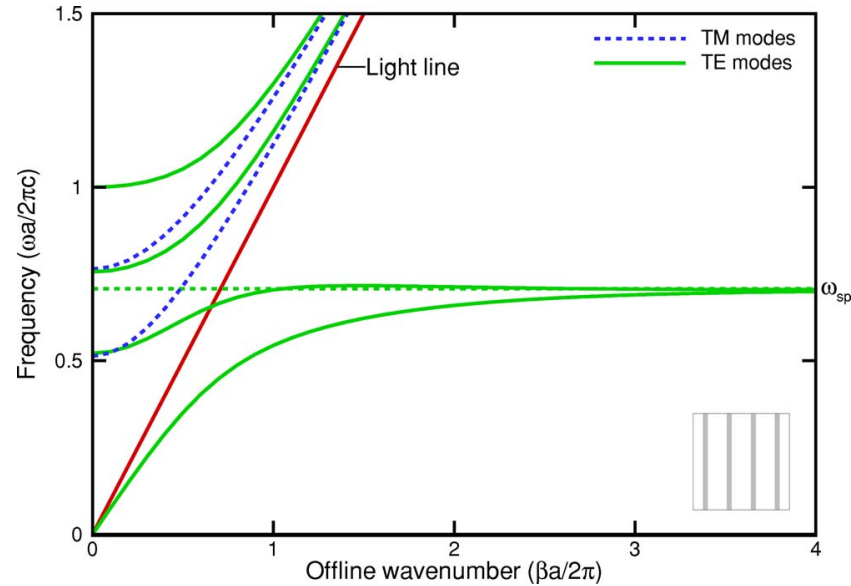

FIG. 2. (Color online) The dispersion relation at $k a / 2 \pi=0.5$ for a one-dimensional metallic layered structure [Fig. 1(a)] of thickness $t / a=0.2$ with $\omega_{p} a / 2 \pi c=1$.

\section{A. One-dimensional layered structures}

For the one-dimensional structures [Fig. 1(a)], there are two surface plasmon modes in the TE bands. One has a lower frequency with odd symmetry, and the other has a higher frequency with even symmetry. However, they eventually converge to the same surface plasma frequency $\omega_{s p}$ at large off-line wave numbers $\beta$, where $\beta$ is the wave number parallel to the metal surface. Figure 2 shows the dispersion relation at $k a / 2 \pi=0.5$ for the thickness ratio $t / a=0.2$ and $\omega_{p} a / 2 \pi c=1$. Splitting of the modes comes from the interaction of surface plasmon polaritons on both sides of the metal as well as the dielectric. The mode with even symmetry has a higher frequency because the mode structure has a larger area that effectively corresponds to a larger energy. For a very thin structure, convergence of surface plasmon modes is slow. This is due to the effective interaction of the modes from both sides of the metal, which lifts the degeneracy. Figure 3 shows the dispersion relation for thickness $t / a$ $=0.1$. In a range of medium fractions of the metal, convergence of surface plasmon modes becomes faster. However,

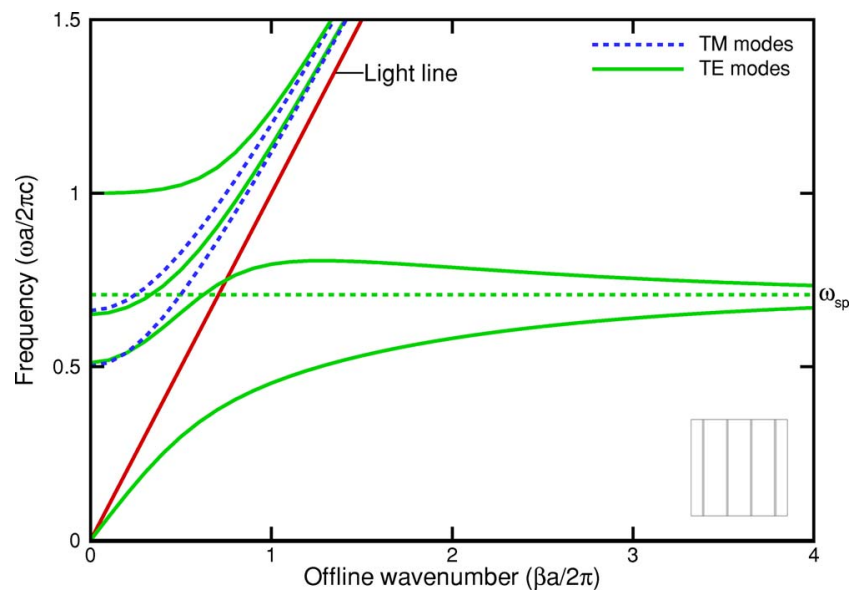

FIG. 3. (Color online) The dispersion relation at $k a / 2 \pi=0.5$ for a one-dimensional metallic layered structure [Fig. 1(a)] of thickness $t / a=0.1$ with $\omega_{p} a / 2 \pi c=1$. 


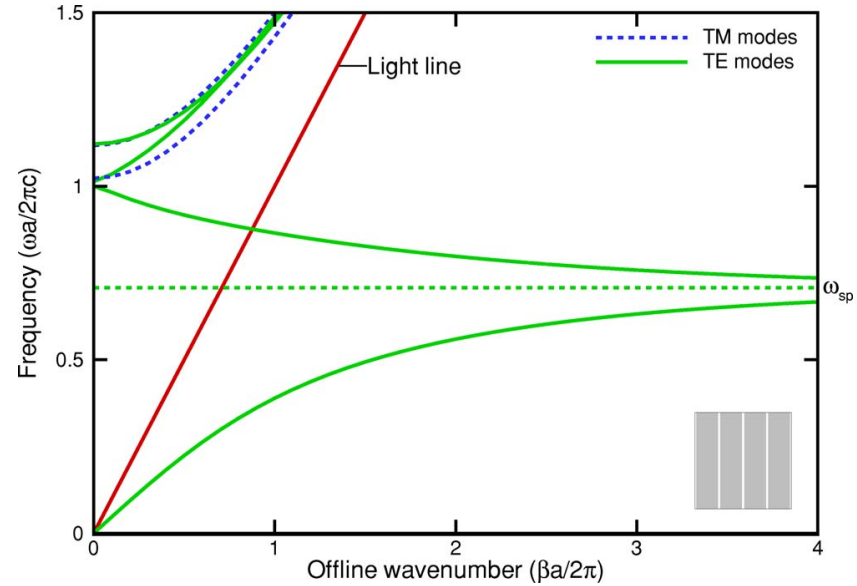

FIG. 4. (Color online) The dispersion relation at $k a / 2 \pi=0.5$ for a one-dimensional metallic layered structure [Fig. 1(a)] of thickness $t / a=0.9$ with $\omega_{p} a / 2 \pi c=1$.

for a very high filling fraction, convergence is slow again, for the degeneracy is also lifted by an effective interaction of the modes from both sides of the dielectric. Figure 4 shows the dispersion relation for thickness $t / a=0.9$. In the meanwhile, the second TE branch has a higher frequency that approaches the bulk plasma frequency $\omega_{p}$ at zero off-line wave number. This is reasonable for the whole lattice is almost filled with the metal. Another important fact in Fig. 4 is the negative group velocity for the second TE branch, which occurs as the dielectric portion becomes sufficiently small. This is consistent with the property of left handedness for the waveguide stack in Ref. [24], which serves as an approach to making a material with a negative index of refraction.

\section{B. Surface plasmon modes}

Surface plasmon modes are eigenmodes of surface plasmon polaritons bound by some constraints in the structure. In this study, the periodicity provides such a constraint, which is also a mechanism to excite surface plasmon modes. It is known that

$$
\omega_{s p}=\frac{\omega_{p}}{\sqrt{1+\varepsilon_{d}}}
$$

is the asymptotic surface plasma frequency at the large- $k$ limit of the dispersion relation for a metallic flat surface [25]. It is also a good approximation for several two-dimensional structures $[13,14]$. Typically, these modes are widely extended through the interface between the dielectric and metal, which are identified as surface plasmon polaritons. The curved or edged interface does not change the frequency of surface plasmon polaritons. Figure 5 shows the $H$ field in magnitude for three typical SPP modes at the point $\Gamma$ near the surface plasma frequency $\omega_{s p}$ for a square array of square metallic cylinders of half width $w / a=0.3$ with $\omega_{p} a / 2 \pi c=1$. Note that in all the plots of the TE eigenmodes, the $H$ field is normalized to have maximum unity-that is, $|H|_{\max }=1$. The computed results show very localized patterns near the interface as they should have. In some extreme cases, the surface plasmon mode may be as sharp as a knife edge residing on the interface. With the interfacial operator approach, it only takes a few points to resolve this feature.

Here, the spatial extension of surface plasmon modes can be estimated by the skin depths at both sides of the metallic flat surface [25]:

$$
\delta_{d}=\frac{\lambda}{2 \pi}\left(\frac{\varepsilon_{d}+\varepsilon_{m}}{\varepsilon_{d}^{2}}\right)^{1 / 2}, \quad \delta_{m}=\frac{\lambda}{2 \pi}\left(\frac{\varepsilon_{d}+\varepsilon_{m}}{\varepsilon_{m}^{2}}\right)^{1 / 2},
$$

where $\lambda$ is the wavelength of light in vacuum, the subscript $d$ denotes the dielectric side, and $m$ denotes the metal side. The skin depths of surface plasmons modes could be very small. As the frequency approaches the surface plasma frequency $\omega_{s p}$, we have $\varepsilon_{d}+\varepsilon_{m} \simeq 0$. In fact, they can be much smaller than the skin depths of the metal, which can be seen from Fig. 5. For comparison, the skin depth for the metal is given by $\delta \equiv 1 / \operatorname{Im}[k]$, where $k=\omega \sqrt{\varepsilon} / c=2 \pi \sqrt{\varepsilon} / \lambda$. Based on the free-electron model (5), it is given as

$$
\delta_{\text {free }}=\frac{\lambda}{2 \pi}\left(\frac{\omega^{2}}{\omega_{p}^{2}-\omega^{2}}\right)^{1 / 2}, \quad \omega<\omega_{p} .
$$

\section{Localized surface plasmons}

On a metallic flat surface, surface plasmons can be widely extended along the surface. For a curved surface, especially for an edged or cornered surface, surface plasmons may be confined in local regions. And for that reason, they are termed as localized surface plasmons [25]. In addition to SPP modes, LSP modes also appear but with somewhat lower frequencies. This is more obvious for structures with sharp edges or corners, such as the square or grid cylinders. Figure 6 shows the $H$ field in magnitude for a typical LSP mode at the point $\Gamma$ for a square array of square metallic cylinders of half width $w / a=0.3$ with $\omega_{p} a / 2 \pi c=1$. Note that the eigenfrequency $\omega a / 2 \pi c=0.5585$ is somewhat lower than the surface plasma frequency $\omega_{s p} a / 2 \pi c=1 / \sqrt{2}$ and the mode structure is concentrated at local areas instead of widely extended along the interface between the dielectric and metal. Besides, the frequencies of LSP modes may change somehow for different geometries, and the bandwidths are a little bit larger than those for SPP modes. However, there is no clear-cut distinction between the modes of LSP and SPP. They belong to different branches of the eigenmodes, possessing the same coupling mechanism but to different degrees. Discussions of localized surface plasmons-in particular, on grating structures — can be found in Refs. [26-28].

In order to measure the degree of localized behavior of surface plasmon modes, we define an extending factor $f_{\text {ext }}$ as follows:

$$
f_{\text {ext }}=\frac{A^{\prime}}{A}=\frac{L^{\prime}}{L},
$$

where $L$ is the total length of the interface and $L^{\prime}$ is the length of the portion of the interface where the magnitude of the $H$ field exceeds a reference value-for example, 0.5 and $A$ and $A^{\prime}$ are the corresponding areas with unit extension along the off-plane direction. For a typical LSP mode, $f_{\text {ext }}$ is 
(a)
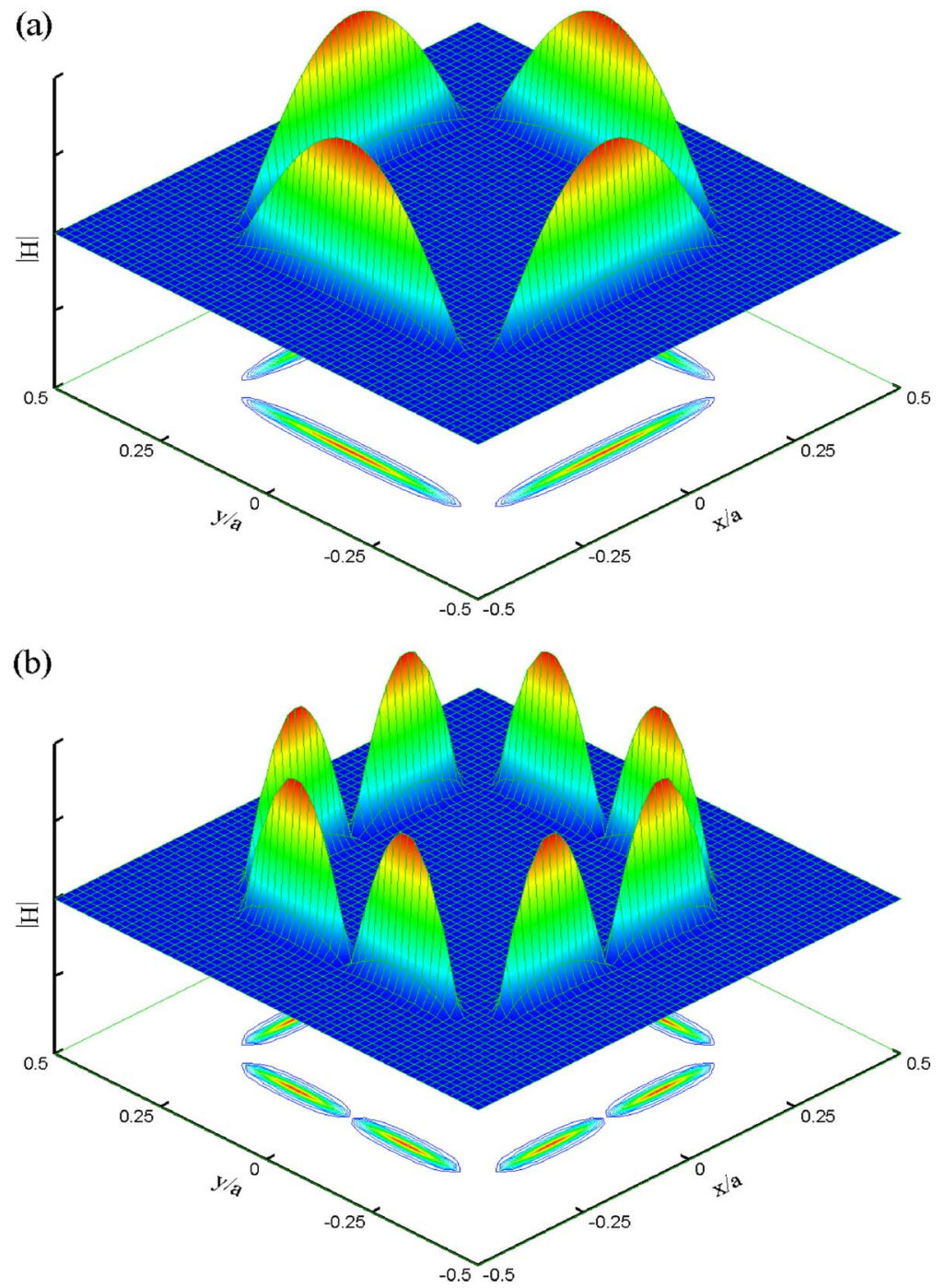

(c)

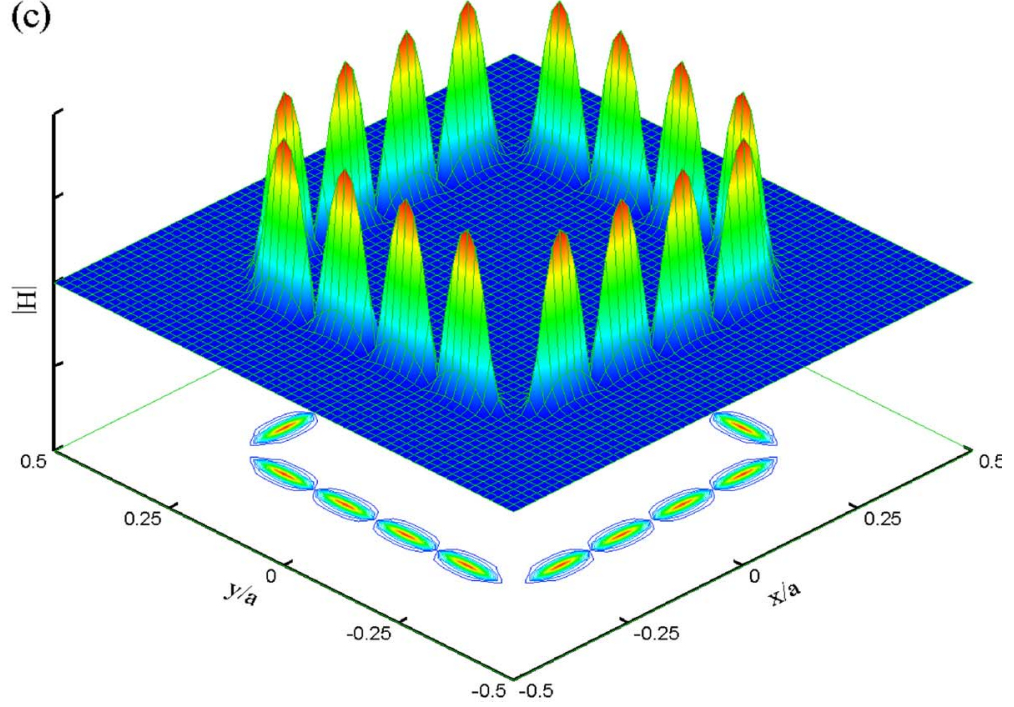

FIG. 5. (Color online) The $H$ field in magnitude for three typical SPP modes with $\omega a / 2 \pi c$ $=0.707$ at the point $\Gamma$ near the surface plasma frequency $\omega_{s p}$ for a square array of square metallic cylinders [Fig. 1(b)] of half width $w / a=0.3$ with $\omega_{p} a / 2 \pi c=1$. usually small. For example, $f_{\text {ext }}=0.33$ for the LSP mode in Fig. 6. On the other hand, $f_{\text {ext }}$ has a larger value for a widely extended SPP mode. In Fig. 5, the SPP mode has an extending factor $f_{\text {ext }}=0.67$.

\section{Surface charges and polarization currents}

Surface charge oscillation is the essential mechanism that supports surface plasmons. For a source-free problem $\left(\rho_{f}\right.$ 


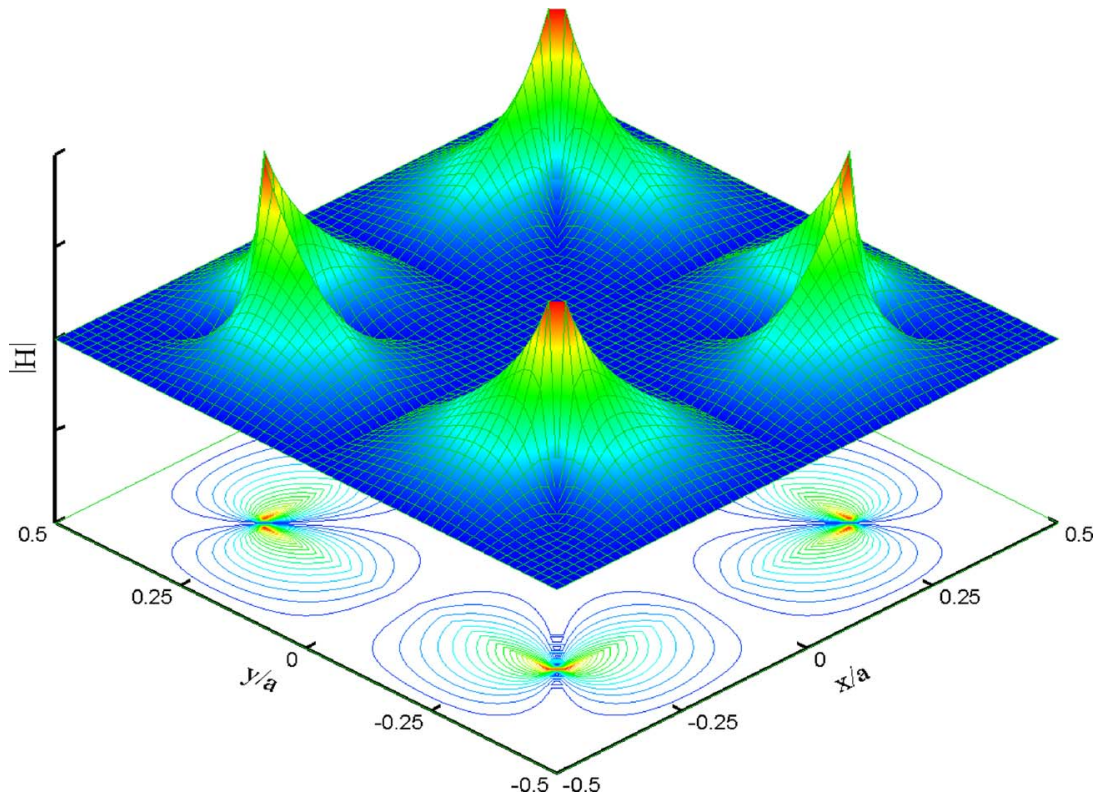

FIG. 6. (Color online) The $H$ field in magnitude for a typical LSP mode with $\omega a / 2 \pi c=0.5585$ at the point $\Gamma$ for a square array of square metallic cylinders [Fig. 1(b)] of half width $w / a=0.3$ with $\omega_{p} a / 2 \pi c=1$.

$=0$ ), surface charges come from discontinuity of the transverse electric field across the interface between the dielectric and metal. From Gauss's law, we have $\rho_{b}=\rho=\varepsilon_{0} \boldsymbol{\nabla} \cdot \mathbf{E}$. For the TE modes, in particular, the transverse electric field components can be obtained from the $H$ field,

$$
E_{x}=\frac{i}{\omega \varepsilon} \frac{\partial H_{z}}{\partial y}, \quad E_{y}=-\frac{i}{\omega \varepsilon} \frac{\partial H_{z}}{\partial x} .
$$

Therefore, we have

$$
\rho_{b}=\frac{i \varepsilon_{0}}{\omega}\left[\frac{\partial}{\partial x}\left(\frac{1}{\varepsilon} \frac{\partial H_{z}}{\partial y}\right)-\frac{\partial}{\partial y}\left(\frac{1}{\varepsilon} \frac{\partial H_{z}}{\partial x}\right)\right] .
$$

Figure 7 shows the transverse electric fields in vectors along with the electric charge strengths in color (online) for a typical surface plasmon mode with $\omega a / 2 \pi c=0.7047$. Note that in the inset the electric field lines flow along the interface, whose nodal points $\left(E_{x}=0\right)$ located at the points where variation of the $H$ field along the interface (the $x$ direction) attains

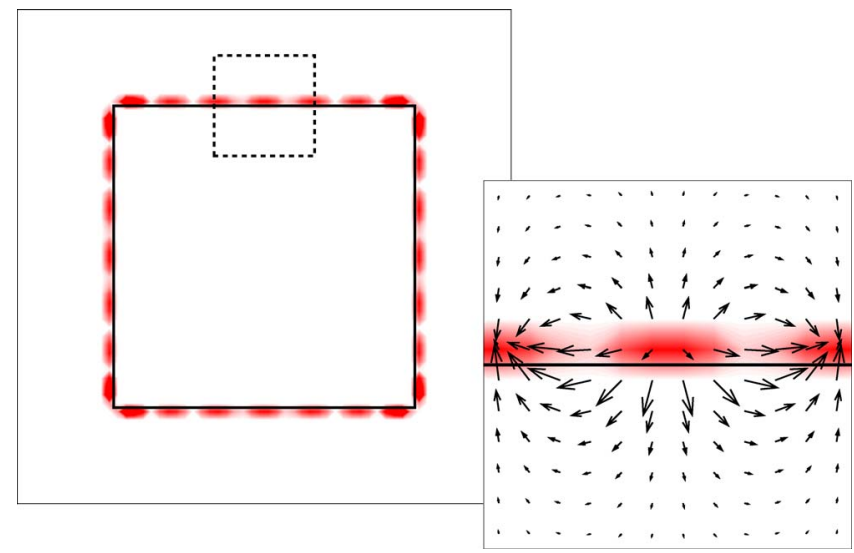

FIG. 7. (Color online) The transverse electric fields in vectors (the inset) along with the electric charge strengths for a typical surface plasmon mode with $\omega a / 2 \pi c=0.7047$. its local maximum value. It is also obvious from the above equation that in the strict insides of the dielectric and metal, the electric charges are identically zero. Therefore, all charges appear on the metal-dielectric interface.

The linear motion of the charges in the metal causes the polarization currents $\mathbf{J}_{p}$ when the polarization field $\mathbf{P}$ changes. According to the free-electron model (5), we have

$$
\mathbf{P}=-\frac{\omega_{p}^{2}}{\omega^{2}} \mathbf{E}, \quad \mathbf{J}_{p}=\frac{i \omega_{p}^{2}}{\omega} \mathbf{E}
$$

Figure 8 shows the polarization currents in vectors and their strengths in color (online) for the same surface plasmon mode in Fig. 7. Note that the polarization currents, flowing in the inside of the metal, also concentrate on the interface and are $90^{\circ}$ out of phase to the electric fields, as compared to Fig. 7.

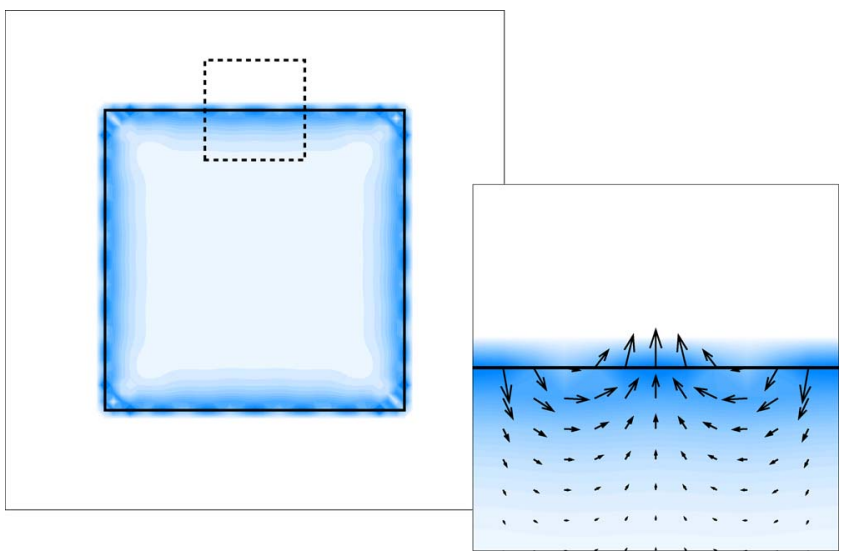

FIG. 8. (Color online) The polarization currents in vectors (the inset) and their strengths for a typical surface plasmon mode with $\omega a / 2 \pi c=0.7047$. 


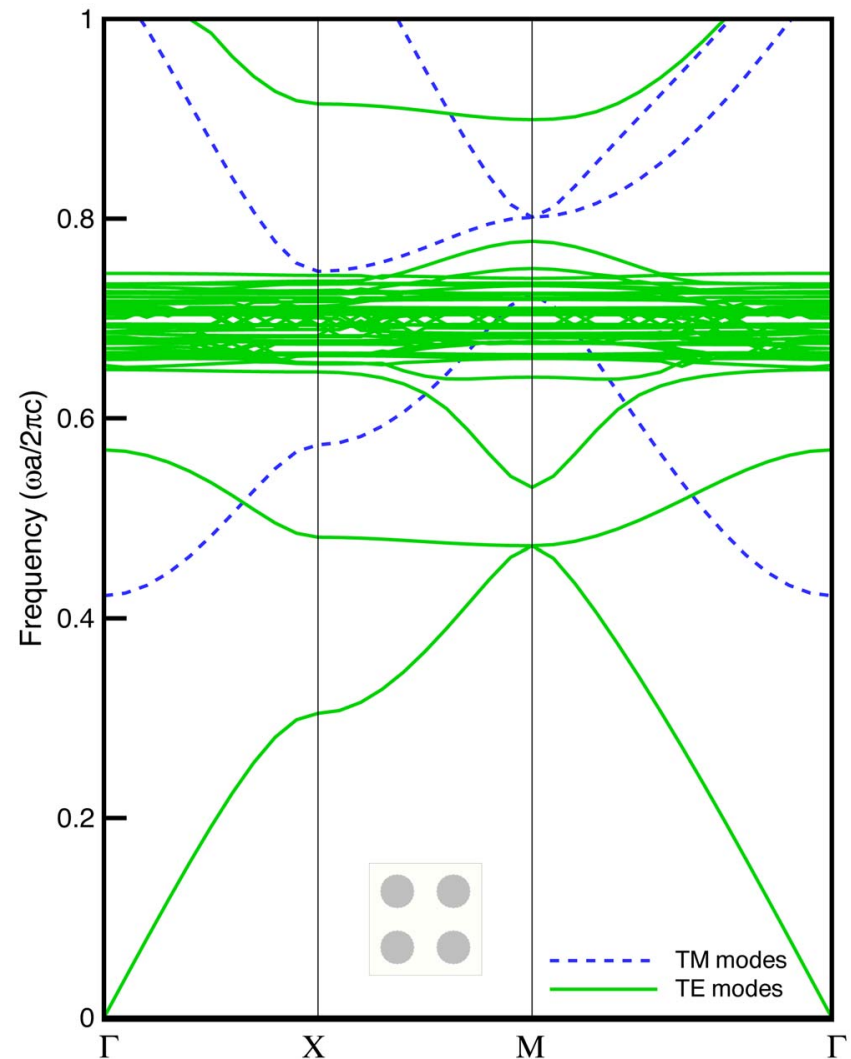

FIG. 9. (Color online) The band structure for a square array of circular metallic cylinders [Fig. 1(c)] of radius $r / a=0.3$ with $\omega_{p} a / 2 \pi c=1$.

\section{E. Band flattening}

The most distinguished feature of band structure for plasmonic crystals is the flatten bands [12,29] associated with SPP modes of small bandwidths, which is due to strong electron-photon coupling that reduces the band dispersion [30]. The band flattening also indicates that massive surface plasmon polaritons are difficult to propagate in the metal. Figure 9 shows the band structure for a square array of circular metallic cylinders of radius $r / a=0.3$ with $\omega_{p} a / 2 \pi c$ $=1$. On the one hand, they are nearly dispersionless and intensively gathered around the surface plasma frequency $\omega_{s p}$. On the other hand, they are highly degenerate in nature. A very high density of states around $\omega_{s p}$ gives rise to strong field enhancements. It seems that variations of the $H$ field along the interface between the dielectric and metal do not alter the value of the eigenfrequency and the interface can sustain as many stationary modes as it could. Consequently, it is expected that there is an infinite number of surface plasmon modes around $\omega_{s p}$, based on the free-electron model or Drude model [13]. This can be addressed further in two aspects. First, the computed results show that the more field points are placed on the interface, the more stationary modes around $\omega_{s p}$ appear. They have almost the same frequency, but with different variations along the interface. The number of stationary modes increases linearly with the grid resolution at the interface. Second, the resonant states of a single metallic circular cylinder also show that there are infinite states

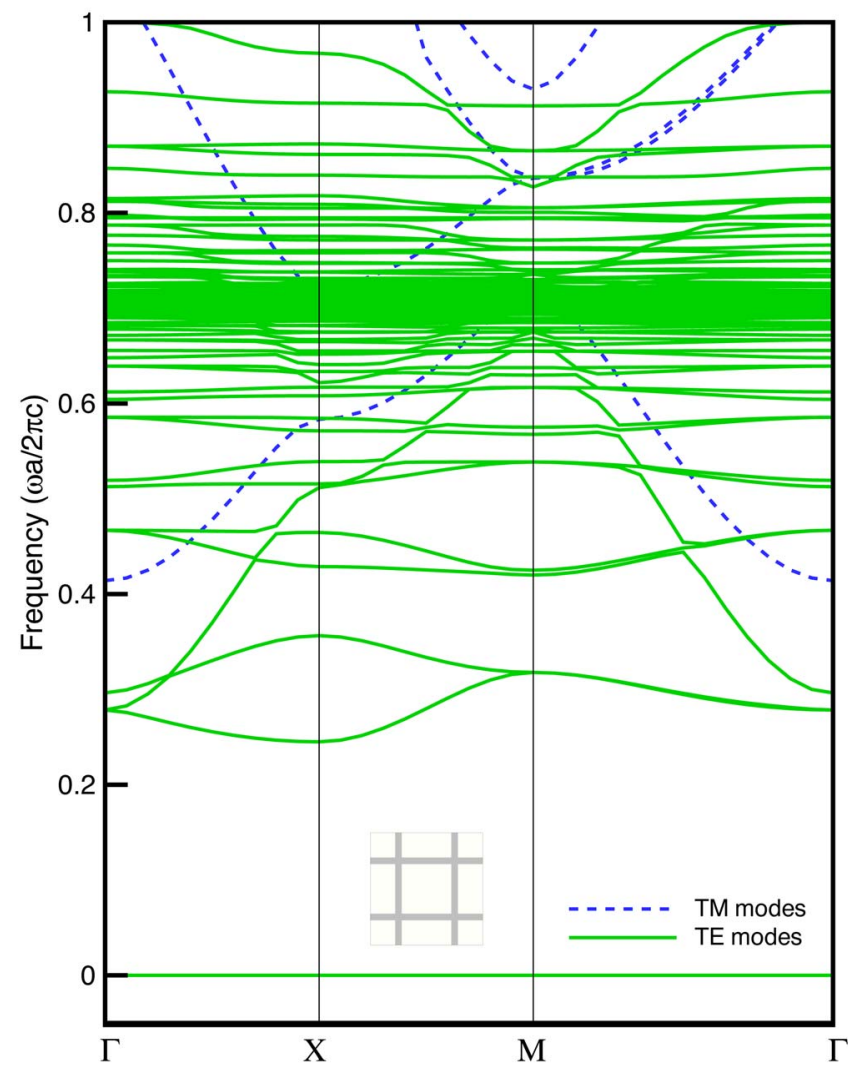

FIG. 10. (Color online) The band structure for a square array of grid metallic cylinders [Fig. 1(d)] of thickness $t / a=0.1$ with $\omega_{p} a / 2 \pi c=1$.

that converge to the same frequency $\omega_{s p}$. Based on the description of linear combination of atomic orbitals (LCAO), it is a fairly accurate approximation to surface plasmon modes [13].

The highly degenerate nature and infinite number of surface plasmon modes can be further explained through the Rayleigh quotient for the operator in the eigensystem (2),

$$
R_{H}=\frac{\langle H, \mathcal{L} H\rangle}{\langle H, H\rangle} .
$$

It is known that the eigenfrequency corresponds to minimization of the Rayleigh quotient under a constraint that the corresponding eigenfunction be orthogonal to all previously obtained eigenfunctions. Substituting the interfacial operator for $\mathcal{L}$ in Eq. (25) along with the Bloch condition (4), we obtain

$$
R_{H}=\frac{\int_{V_{d}} \frac{1}{\varepsilon_{d}}|\nabla H|^{2} d \tau+\int_{V_{m}}\left[|\nabla H|^{2}+\left(\omega_{p} / c\right)^{2}|H|^{2}\right] d \tau}{\int_{V_{\text {cell }}}|H|^{2} d \tau},
$$

where $V_{d}$ and $V_{m}$ denote the volumes of the dielectric and metal, respectively, and $V_{\text {cell }}$ the volume of the unit cell. From Eq. (26) we know that variations in the tangential di- 


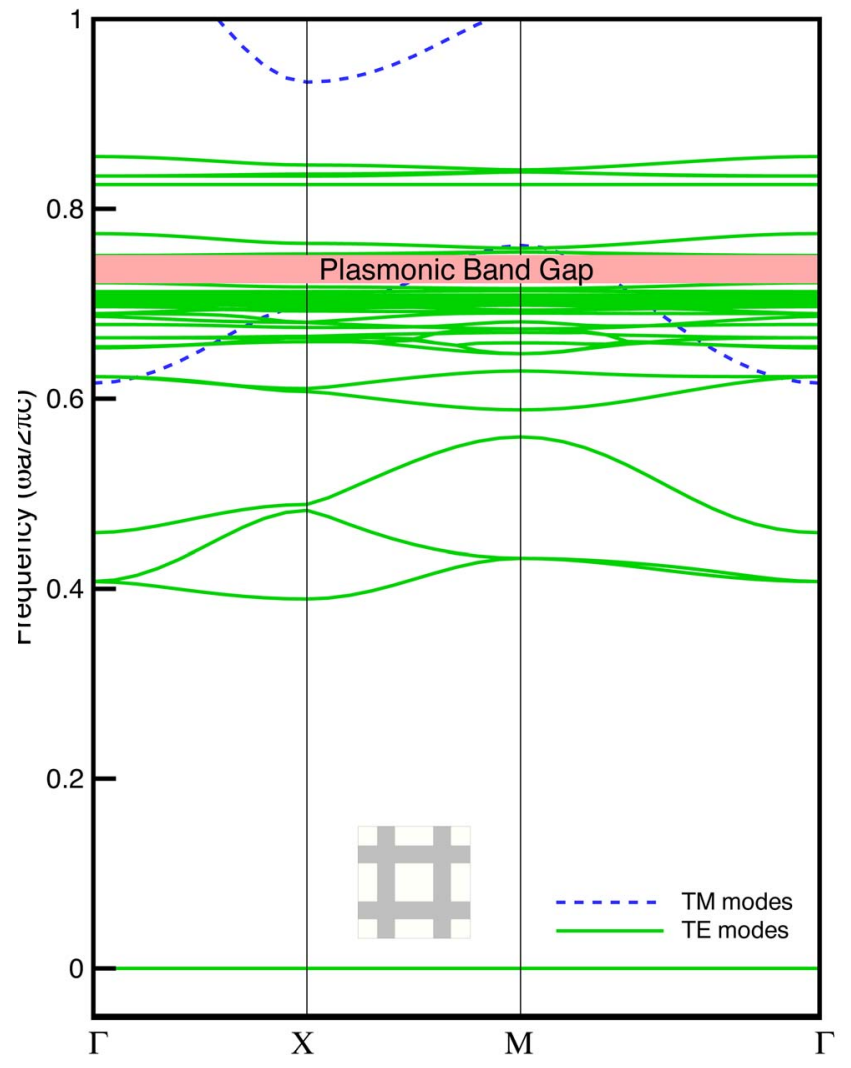

FIG. 11. (Color online) The band structure for a square array of grid metallic cylinders [Fig. 1(d)] of thickness $t / a=0.3$ with $\omega_{p} a / 2 \pi c=1$.

rection along the interface will change neither the value of the Rayleigh quotient nor the eigenfrequency. There can be as many modes as possible if the variation of the field in the normal direction to the interface remains unchanged. This is also verified in Fig. 5, that the typical feature of the SPP modes is similar except a different variation along the interface. However, the eigenfrequency is almost identical. Numerically, the modes can be resolved only when the grid resolution is fine enough to tell the tangential variation. However, this infinite degeneracy of the eigenmodes may be removed if a more realistic model is considered, by taking into account, for instance, the interband transition and the spatial dispersion [13].

\section{F. Band broadening}

In addition to the band flattening, we see another feature of SPP modes - that is, the band broadening. For thin metallic structures, SPP modes become more widely spread around the surface plasma frequency $\omega_{s p}$. The physical origin of this broadening could be explained as an effective mode interaction due to the geometry of the interface. In particular, for thin grid structures, the interaction from both the normal and lateral directions of the interface further lifts the degeneracy, making the bands spread even more widely. Figure 10 shows the band structure for a square array of grid metallic cylinders of thickness $t / a=0.1$ with $\omega_{p} a / 2 \pi c=1$. On the other hand, for thick grid structures, there may be a balance

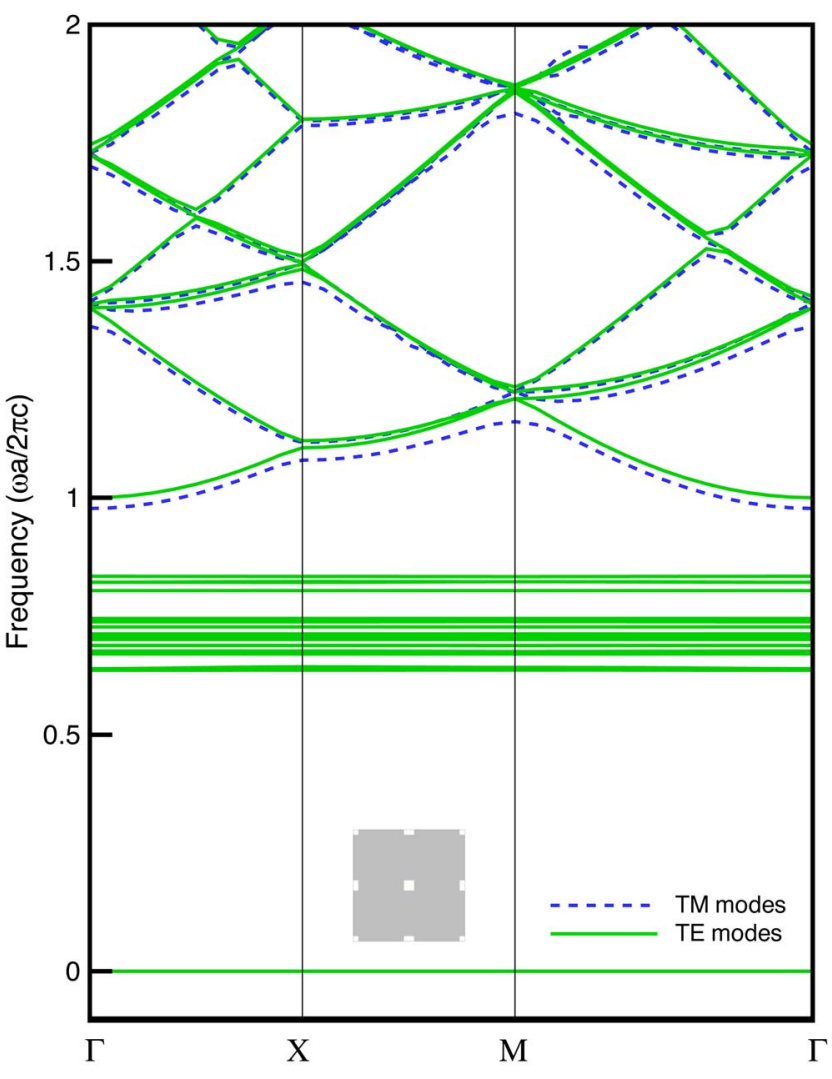

FIG. 12. (Color online) The band structure for a square array of grid metallic cylinders [Fig. 1(d)] of thickness $t / a=0.8$ with $\omega_{p} a / 2 \pi c=1$.

between band flattening and band broadening. As a result, a plasmonic band gap between surface plasmon modes may occur around $\omega_{s p}$, although it is usually small. Figure 11 shows the band structure for a square array of grid metallic cylinders of thickness $t / a=0.3$ with $\omega_{p} a / 2 \pi c=1$. A plasmonic band gap with a gap-to-midgap ratio of $3.9 \%$ and midgap frequency of 0.738 is shown in the figure.

\section{G. Cutoff behavior}

For dielectric structures, there is an acoustic branch which is linear at small wave vectors with zero eigenfrequency at the point $\Gamma$ for both the TM and TE modes. However, for structures that contain metallic components either modeled as perfectly conductors [31] or dispersive metals [15], this branch may disappear in the TM modes due to the cutoff behavior. In other words, zero-frequency solutions do not exist for all wave vectors. For one-dimensional layered structures, the greater the filling fraction of the metal, the smaller the required threshold for the occurrence of the cutoff frequency [11]. Also, the higher the fraction of the metal, the larger the cutoff frequency. The cutoff frequency significantly modifies the band structure for the TM modes by introducing a zeroth-order band gap [31]. When the cutoff frequency is large enough, the full band gap is solely determined by the TE bands. A comparison of Figs. 10 and 11 shows that the cutoff frequency for a thick structure is larger than for a thin structure. Besides, as the fraction of 


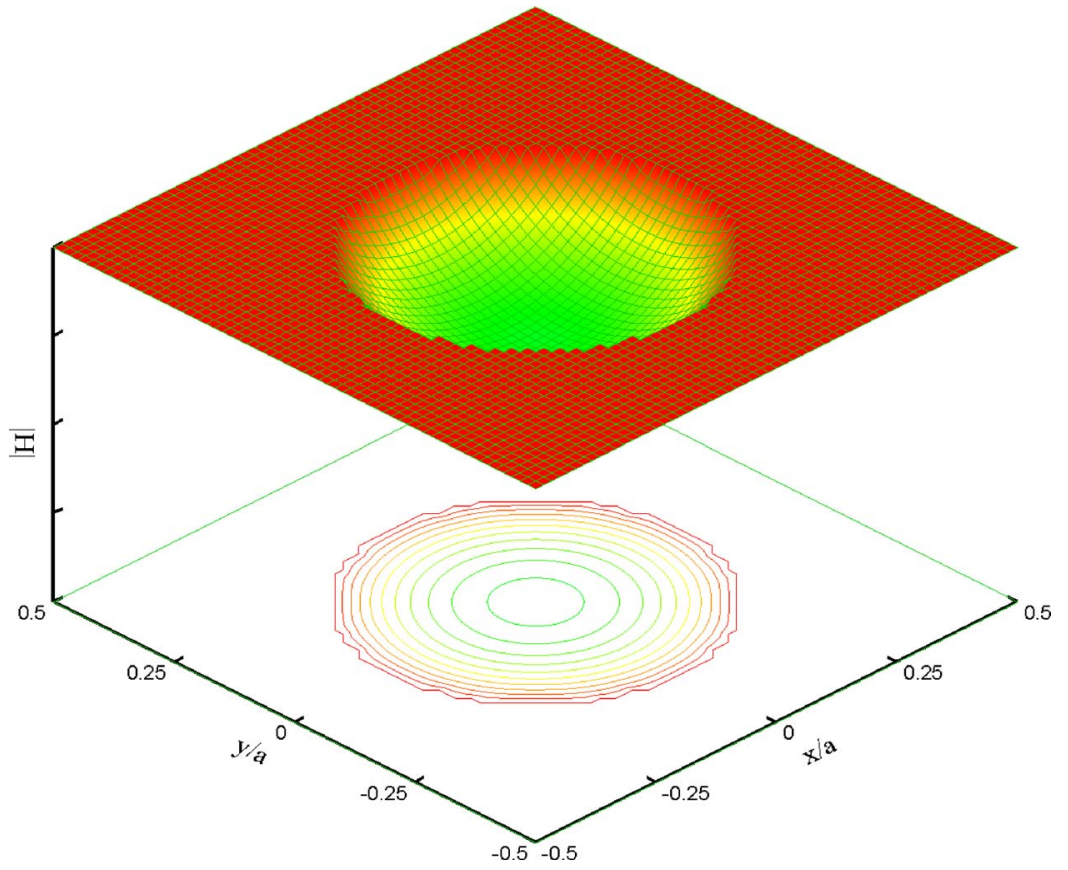

FIG. 13. (Color online) The static mode at the point $\Gamma$ for a square array of circular metallic cylinders [Fig. 1(c)] of radius $r / a=0.3$ with $\omega_{p} a / 2 \pi c=1$. metallic components increases, the TE bands below $\omega_{p}$ tend to be dispersionless. For very high fractions of metals, all the TM bands may lie above $\omega_{p}$ and coincide with the TE bands there, while the other TE bands below $\omega_{p}$ are reduced to a few flatbands. Figure 12 shows the band structure for a square array of grid metallic cylinders of thickness $t / a=0.8$ with $\omega_{p} a / 2 \pi c=1$. On the other hand, for the same metal fraction, dispersive metals with a larger plasma frequency $\omega_{p}$ also exhibit a larger cutoff frequency [15]. Both cases indicate that the density of free electrons contained in the metal is responsible for the cutoff behavior. In another aspect, the cutoff frequency is also related to the energy stored in the electromagnetic field. The integrand $\omega_{p}^{2}|H|^{2}$ in the Rayleigh quotient (26) indicates that the free electrons in metallic materials are likely to raise the energy of the field, which is in contrast to the situation in dielectric materials, where the field tends to concentrate on the high- $\varepsilon$ region in order to lower its frequency. This can be made clear by the energy density $u$ of the electromagnetic field for dispersive materials [32],

$$
u=\frac{1}{2} \operatorname{Re}\left[\frac{d(\omega \varepsilon)}{d \omega}\right] E^{2}+\frac{1}{2} \operatorname{Re}\left[\frac{d(\omega \mu)}{d \omega}\right] H^{2} .
$$

Based on the free-electron model (5), we have

$$
u=\frac{1}{2} \varepsilon_{0}\left(1+\frac{\omega_{p}^{2}}{\omega^{2}}\right) E^{2}+\frac{1}{2} \mu_{0} H^{2},
$$

which reveals that the energy may increase with increasing the plasma frequency.

It is natural for us to consider that the cutoff behavior is analogous to that in conventional waveguides. However, the origin of the cutoff behavior in the TM modes for the metallic structure is very different from that of conventional waveguides. In the latter, the cutoff frequency originates from sustenance of the transverse oscillations [33], while in the former, the electric fields are identically zero inside the perfect conductors, or exponentially decay inside the dispersive metals. In particular, we consider the TM modes at the symmetric point $\Gamma$. If the zero-frequency solution (without a cutoff frequency) is allowed in the structure, the $E$ field should be constant everywhere outside the metal. By the

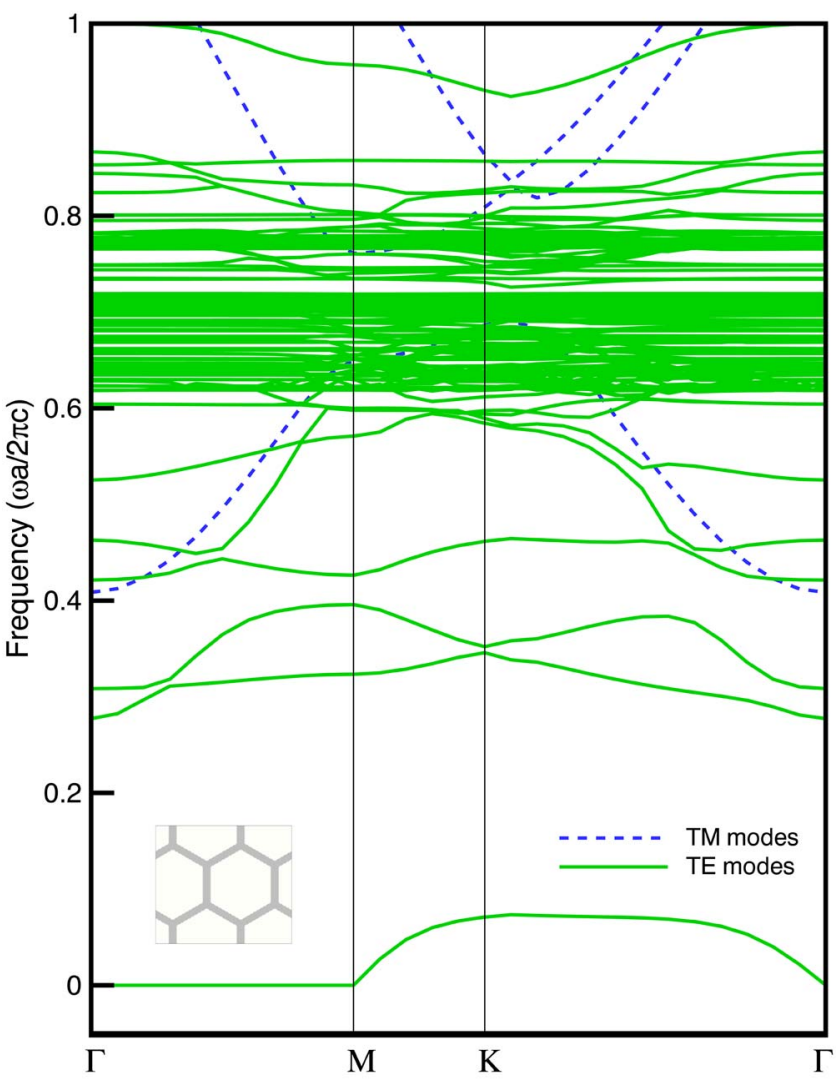

FIG. 14. (Color online) The band structure for a hexagonal array of grid metallic cylinders of thickness $t / a=0.1$ with $\omega_{p} a / 2 \pi c=1$. 


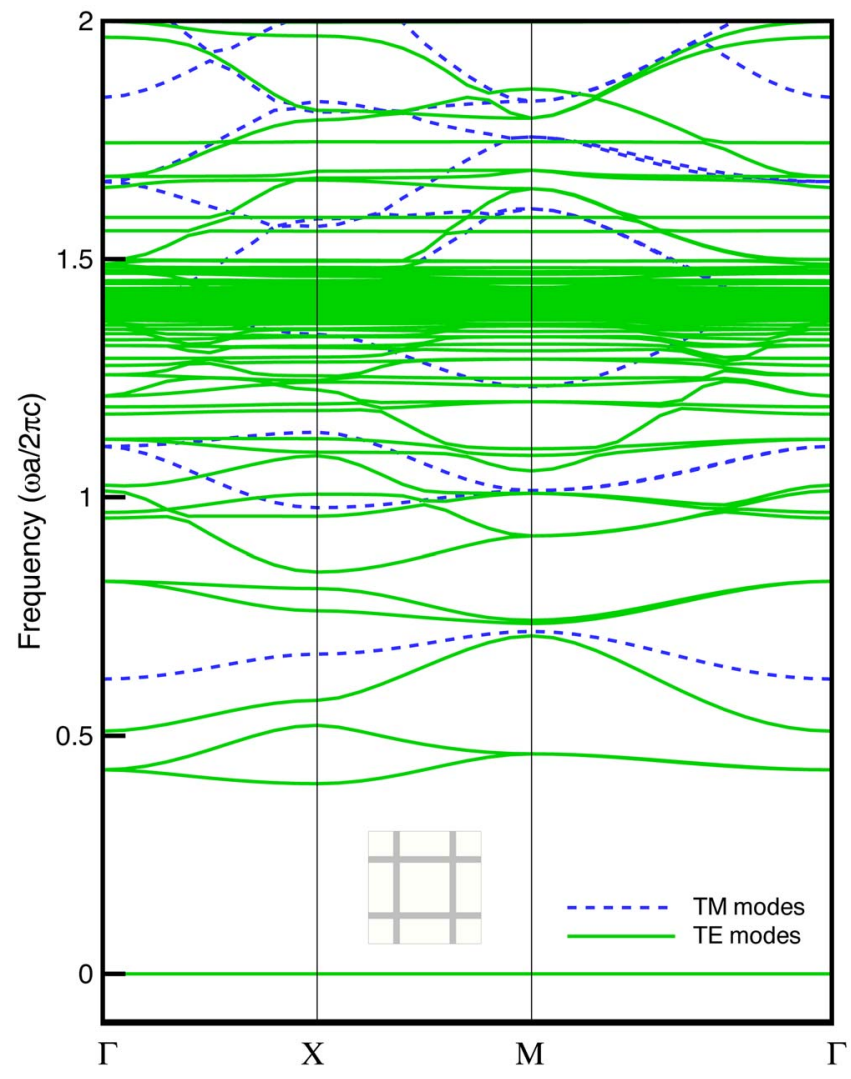

FIG. 15. (Color online) The band structure for a square array of grid metallic cylinders [Fig. 1(d)] of thickness $t / a=0.1$ with $\omega_{p} a / 2 \pi c=2$.

continuity of the $E$ field at the metal boundary for perfect conductors $\left([E]_{S}=0\right)$, this constant must be zero, resulting in a trivial solution. For dispersive metals, another continuity condition of the $E$ field at the metal boundary $\left([\partial E / \partial n]_{S}\right.$ $=0$ ) excludes the possibility of a constant solution outside the metal and exponentially decay inside. In either case, the TM modes with zero frequency do not exist, making the appearance of the cutoff frequency.

On the other hand, the cutoff behavior is not observed in the TE modes for metallic structures. This is because constant solutions outside the metal (and zero inside) with zero frequency are allowed in the structure without violating the boundary condition for perfect conductors $\left([\partial H / \partial n]_{S}=0\right)$. For dispersive metals, the zero-frequency solution is also valid, for the interface condition (12) allows a discontinuity in the normal derivative of the $H$ field across the metal boundary, which is necessary for a solution to be constant outside the metal and exponentially decay inside.

\section{H. Static modes}

Static modes are zero-frequency solutions mentioned in the preceding subsection. Figure 13 shows the static mode at the point $\Gamma$ for a square array of circular metallic cylinders of radius $r / a=0.3$ with $\omega_{p} a / 2 \pi c=1$. They are eigenmodes correspond to the long-wavelength limit, also the electrostatic field for the TM modes or the magnetostatic field for the TE modes. In dielectric structures, static modes appear in both the TM and TE modes. For metallic structures, however, only the TE modes sustain static modes. Besides, in isolated metallic structures, static modes appear only at $\mathbf{k}=0$ (point $\Gamma)$, but for connected structures, static modes may appear at other and even all wave vectors. This is because connectivity of the structure provides a degree of freedom to let static modes accommodate themselves in the metal to any value of $\mathbf{k}$ in the direction perpendicular to the connectivity, without violating the Bloch condition at the cell boundary. For example, the band structure for a square array of metallic grid cylinders shown in Figs. 10-12 has a zero-frequency branch in the TE bands along the $\Gamma-X-M-\Gamma$ path in the first Brillouin zone. We also observe the same feature in one-dimensional layered structures. In fact, this is the extreme case of reso-

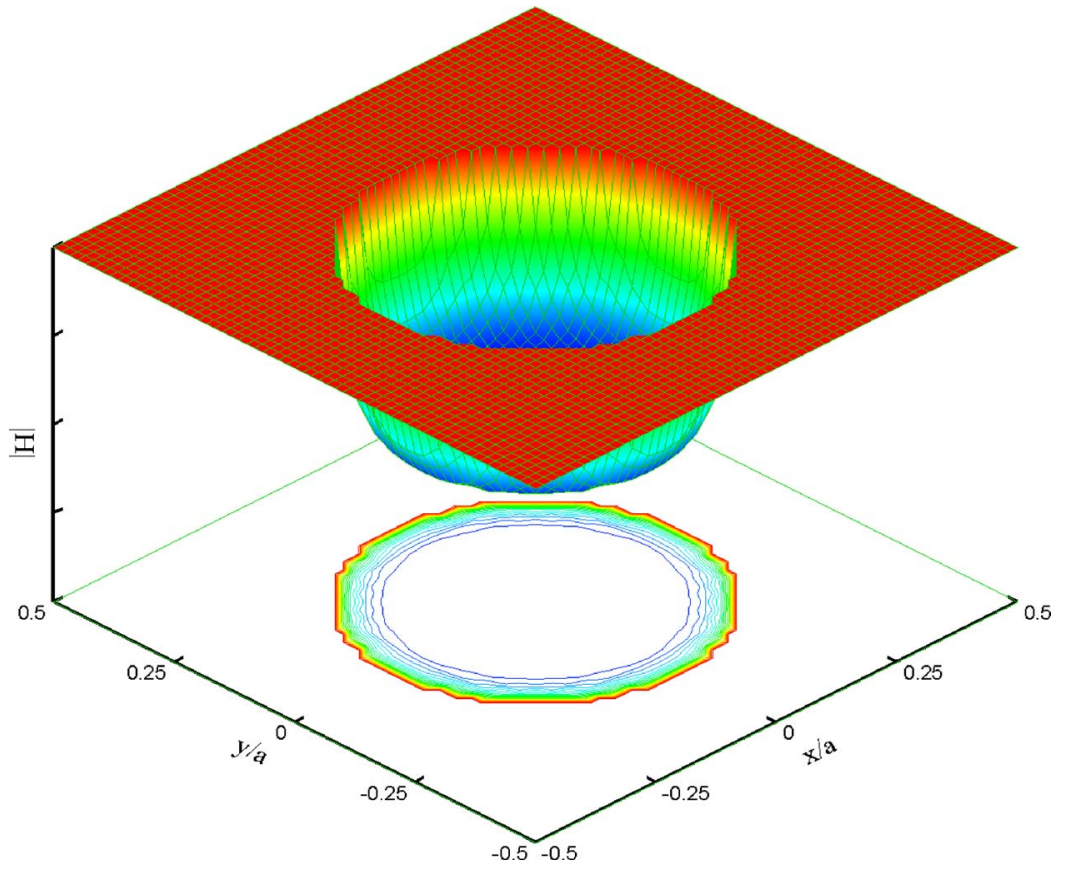

FIG. 16. (Color online) The static mode at the point $\Gamma$ for a square array of circular cylinders [Fig. 1(c)] of radius $r / a=0.3$ with $\omega_{p} a / 2 \pi c=8$. 


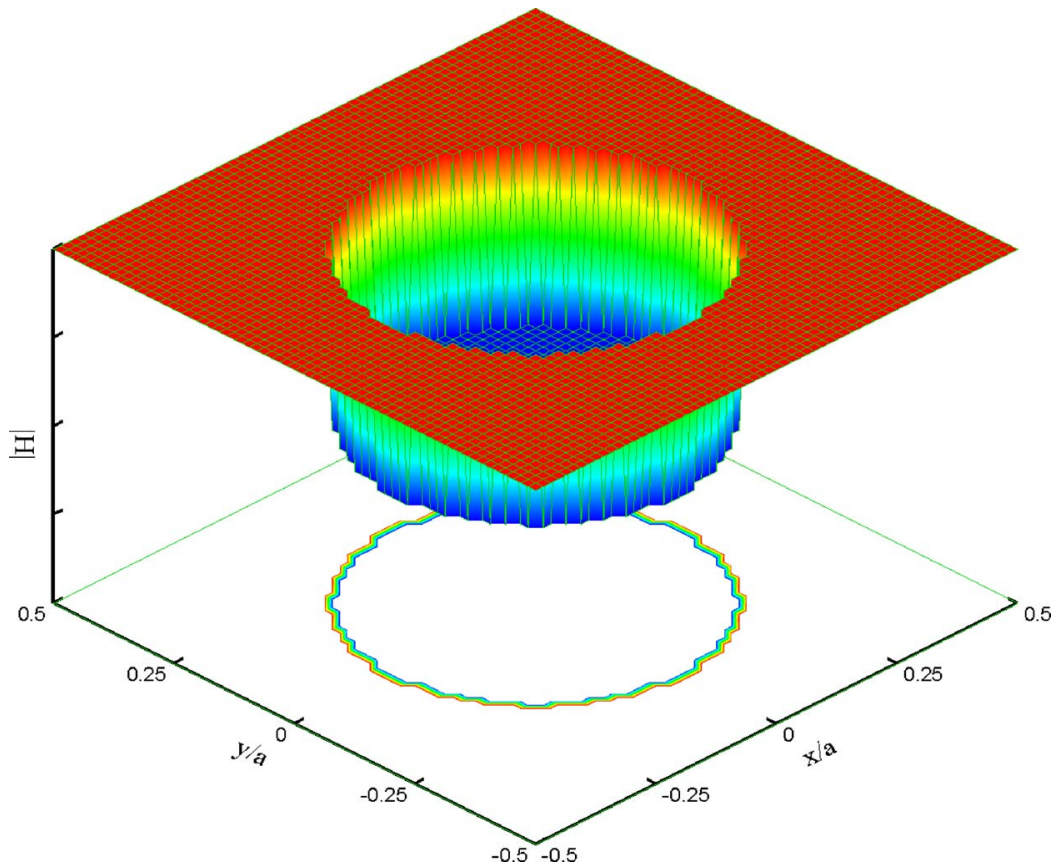

FIG. 17. (Color online) The static mode at the point $\Gamma$ for a square array of circular perfectly conducting cylinders [Fig. 1(c)] of radius $r / a$ $=0.3$. nant modes in the cavities [33]. They are standing waves confined in the metal, which are independent of the wave vectors. For isolated structures, this does not occur.

If this phenomenon is true in both directions of lattice translation-for example, a square array of metallic grid cylinders - then a branch merely consisting of static modes is formed. As a result, the first branch is a straight zero band and a very large band gap is opened and solely determined by the second branch. This is demonstrated in the band structure for a square array of metallic grid cylinders in Fig. 10. In a sense, this band gap resembles the zeroth-order band gap caused by the cutoff frequency in the TM modes. This observation also applies to the case of perfect conductors, where all branches are indeed straight lines [34]. On the other hand, if the structure exhibits connectivity in only one lattice direction, then a partial zero band is observed. For example, a hexagonal array of metallic grid cylinders will have a zero-frequency branch in the TE bands along the $\Gamma-M$ path in the first Brillouin zone. Figure 14 shows the band structure for a hexagonal array of grid metallic cylinders of thickness $t / a=0.1$ with $\omega_{p} a / 2 \pi c=1$.

\section{Plasma frequency}

The plasma frequency $\omega_{p}$ is the natural frequency of collective oscillation of free electrons. It is a measure for the

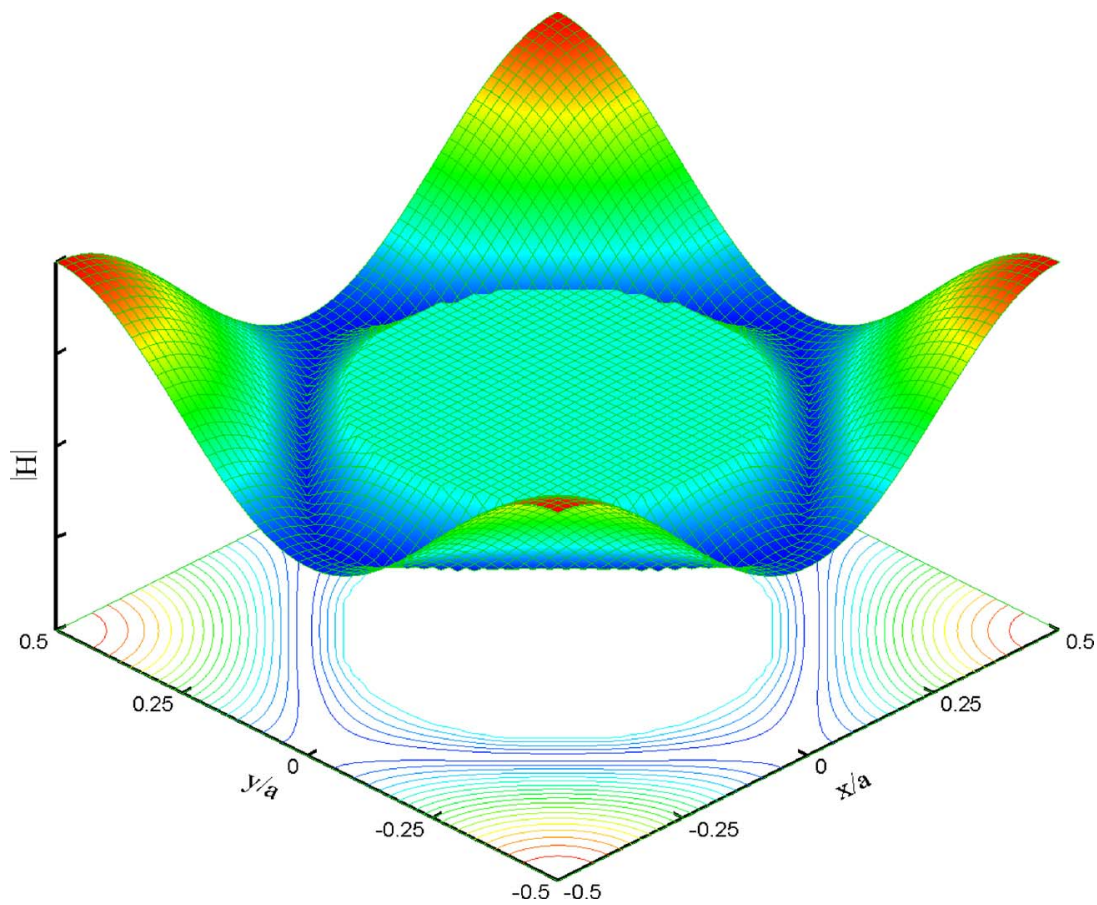

FIG. 18. (Color online) The longitudinal mode at the point $\Gamma$ for a square array of circular metallic cylinders [Fig. 1(c)] of radius $r / a=0.3$ with $\omega_{p} a / 2 \pi c=1$. 


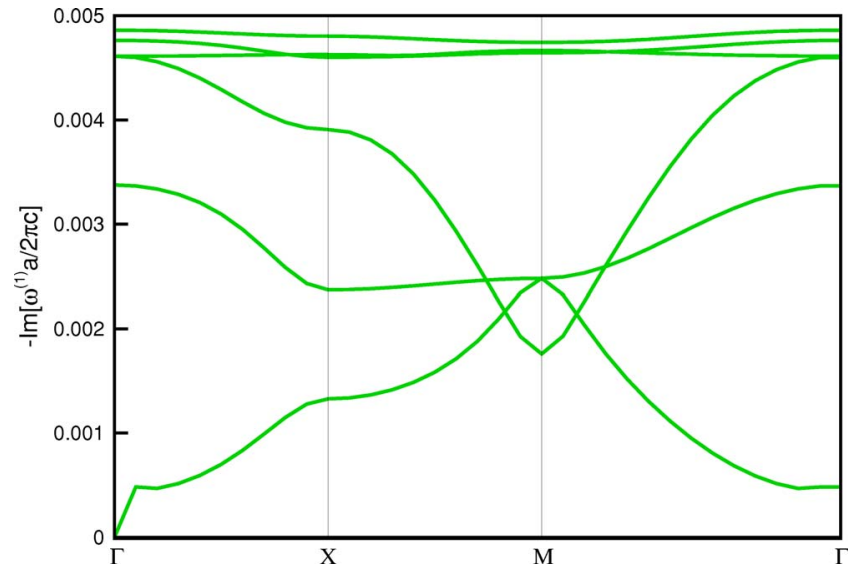

FIG. 19. (Color online) The imaginary part of $\omega_{1}$ of the first few bands for a square array of square metallic cylinders [Fig. 1(b)] of half width $w / a=0.3$ with $\omega_{p} a / 2 \pi c=1$ and $\gamma / \omega_{p}=0.01$

number density of free electrons [17]. The higher the plasma frequency, the more free electrons contained in a metal. Based on the free-electron model (5), the plasma frequency $\omega_{p}$ has several effects on the skin depth of the metal (20), the Rayleigh quotient of the eigensystem (26), the energy density of electromagnetic fields in the metal (28), and most important, the surface plasma frequency $\omega_{s p}$, through relation (18). Besides, the surface plasmon band structures are no longer scale invariant because of the appearance of $\omega_{p}$. That means solutions with different $\omega_{p}$ cannot be scaled with each other. Figure 15 shows the band structure for a square array of grid metallic cylinders of thickness $t / a=0.1$ with $\omega_{p} a / 2 \pi c=2$. Note that the detailed structure is different from the case of $\omega_{p} a / 2 \pi c=1$ in Fig. 10, although the overall pattern is similar. Besides, surface plasma frequency $\omega_{s p}$ switches to a higher value $\omega_{s p} a / 2 \pi c=\sqrt{2}$.

As $\omega_{p}$ increases to a higher value, the energy density of the electromagnetic field (28) becomes larger and the group velocities of surface plasmon polaritons become even smaller. In another aspect, dispersive metals behave more like perfect conductors at large $\omega_{p}$. The transition from dispersive metals to perfect conductors can be illustrated through the changes of static modes. Figure 16 shows the static mode at the point $\Gamma$ for a square array of circular metallic cylinders of radius $r / a=0.3$ with $\omega_{p} a / 2 \pi c=8$. The skin depth (20) for static modes is given by

$$
\delta_{\text {free }}=\frac{c}{\omega_{p}},
$$

where $c$ is the speed of light in vacuum. As $\omega_{p}$ becomes larger, the skin depth becomes smaller and the field more evanescent. In the limiting case where $\omega_{p}$ approaches infinity, dispersive metals behave like perfect conductors. Figure 17 shows the static mode at the point $\Gamma$ for a square array of circular perfectly conducting cylinders of radius $r / a=0.3$. There is an unlimited supply of free electrons [35], and all fields are expelled out from the metal and vanish inside. Consequently, there are no bound states to support surface plasmons. This can also be explained through relation (18) between $\omega_{s p}$ and $\omega_{p}$. When $\omega_{s p}$ approaches infinity, surface plasmon modes with finite frequencies disappear. Nevertheless, surface plasmons may be mimicked on a perfectly conducting surface with a structured array of holes much smaller than the wavelength [36].

\section{J. Longitudinal modes}

The electromagnetic fields are transverse in nature. However, longitudinal modes may exist in a material when the dielectric constant becomes zero. According to the freeelectron model (5), a longitudinal mode exists when its eigenfrequency is equal to the plasma frequency $\omega_{p}$. That also means that oscillation of the electric field coincides with coherent motion of the electrons. Like surface plasmon modes, longitudinal modes appear only in the TE modes, for the transversality condition of the $E$ field $(\boldsymbol{\nabla} \cdot \mathbf{E}=0)$ is always met for the TM modes. However, longitudinal modes are difficult to obtain due to the singularity of the operator in Eq. (2). Nevertheless, with rearrangement of the interfacial operator approach in Eq. (2), based on the free-electron mode (5), the singularity is removed and longitudinal modes can be solved. Figure 18 shows the static mode at the point $\Gamma$ for a square array of circular metallic cylinders of radius $\mathrm{r} / \mathrm{a}$ $=0.3$ with $\omega_{p} a / 2 \pi c=1$. Note that the longitudinal mode is constant in the metal, which is the typical feature of longitudinal oscillation.

Static and longitudinal modes are two unique features in the TE modes for metallic structures. In a sense, they are dual to each other; static modes have constant solutions outside the metal, while longitudinal modes have constants inside. In another aspect-for example, in one-dimensional layered structures - both the static mode and the longitudinal mode eventually converge to two surface plasmon modes at sufficiently large off-line wave numbers. These unique features originate from the fact that the dielectric function lies inside the operator for the TE modes (2). On the other hand, they do not exist in the TM modes because the interface condition for the $E$ field $\left([\partial E / \partial n]_{S}=0\right)$ excludes such solutions. That also means that surface charges are responsible for static and longitudinal modes as they are for surface plasmon modes.

\section{K. Drude damping}

Using the perturbation technique, we can study the effect of Drude damping, provided that the collision frequency $\gamma$ is small compared to the eigenfrequency $\omega$. The imaginary part of the first-order correction $\omega_{1}$ in Eq. (17) gives rise a decay factor $e^{\operatorname{Im}\left[\omega_{1}\right] t}$ of the mode structure in the time domain. Note that $\operatorname{Im}\left[\omega_{1}\right]$ is negative for an $e^{-i \omega t}$ dependance of the fields. Figure 19 shows the imaginary part of $\omega_{1}$ of the first few bands for a square array of square metallic cylinders of half width $w / a=0.3$ with $\omega_{p} a / 2 \pi c=1$ and $\gamma / \omega_{p}=0.01$. In general, $\operatorname{Im}\left[\omega_{1}\right]$ is smaller than the collision frequency $\gamma$ by about one order of magnitude. Even bands have larger dissipation at the zone center, while odd bands have larger dissipation at the band edges. Moreover, the dissipation becomes even smaller for higher bands. 


\section{CONCLUDING REMARKS}

In this paper, we compute surface plasmon modes and band structures for plasmonic crystals based on the freeelectron and Drude models. By using the interfacial operator approach, surface plasmon band structures can be solved directly in the frequency domain. In particular, the introduction of interfacial variables enables resolution of the highly localized nature of surface plasmon modes near the interface of the dielectric and metal. The above two features facilitate greatly investigation of the optical properties of plasmonic crystals. For one-dimensional layered structures, we study the mode splitting and convergence of surface plasmon modes, as well as the negative group velocity for the second TE branch. For two-dimensional problems, we study the typical mode structure of surface plasmon modes, along with identification of SPP and LSP modes. The transverse fields, surface charges, and polarization currents are visualized to study various properties of surface plasmon modes. The localized nature of SPP modes around the surface plasma frequency provides the theoretical basis for plasmonic circuit.

In addition, the band flattening, band broadening, and possible plasmonic band gaps are presented for thin and thick metallic structures. The Rayleigh quotient for the TE modes is employed to understand the highly degenerate nature of surface plasmon modes and the possibility of infinite degrees of degeneracy. The cutoff behavior for TM modes (but not for TE modes) is explained on a unified basis by examining different types of boundary conditions for TM and TE modes and which is further clarified by considering the energy density in the electromagnetic fields for dispersive metals. The band broadening for thin metallic structures allows a wide range of absorption and therefore may find potential applications in solar cells. An interesting discussion has being also devoted to the relationship of the existence of static modes and connectivity of the metal. The related skin depth for the dispersive metal and the transition to the perfect conductor are also discussed. Moreover, longitudinal modes, which appear only in the TE bands, are also obtained in the present study. Finally, the effect of Drude damping is studied by a perturbation technique to the undamped solutions. Nevertheless, in the wide range of energy spectrum, $0-2 \hbar \omega_{p}$, the effect of interband transitions is not negligible $[37,38]$. This proposes a further challenge for the present methodology because the contribution of the interband transitions lacks a simple functional form in frequency. This topic is now under investigation, and the results will be reported elsewhere.

\section{ACKNOWLEDGMENTS}

This work was supported in part by the National Science Council of the Republic of China under Contract Nos. NSC 94-2212-E-002-047 and NSC 94-2212-E-002-076 and the Ministry of Economic Affairs of the Republic of China under Contract No. MOEA 94-EC-17-A-08-S1-0006.
[1] E. Yablonovitch, Phys. Rev. Lett. 58, 2059 (1987).

[2] S. John, Phys. Rev. Lett. 58, 2486 (1987).

[3] I. I. Smolyaninov, W. Atia, and C. C. Davis, Phys. Rev. B 59, 2454 (1999).

[4] G. Shvets and Y. A. Urzhumov, J. Opt. A, Pure Appl. Opt. 7, S23 (2005).

[5] B. Hecht, H. Bielefeldt, L. Novotny, Y. Inouye, and D. W. Pohl, Phys. Rev. Lett. 77, 1889 (1996).

[6] J. Pendry, Science 285, 1687 (1999).

[7] K. Kneipp, Y. Wang, H. Kneipp, L. T. Perelman, I. Itzkan, R. R. Dasari, and M. S. Feld, Phys. Rev. Lett. 78, 1667 (1997).

[8] S. M. Nie and S. R. Emery, Science 275, 1102 (1997).

[9] P. Andrew, S. C. Kitson, and W. L. Barnes, J. Mod. Opt. 44,395 (1997).

[10] M. Westphalen, U. Kreibig, J. Rostalski, H. Luth, and D. Meissner, Sol. Energy Mater. Sol. Cells 61, 97 (2000).

[11] O. Toader and S. John, Phys. Rev. E 70, 046605 (2004).

[12] V. Kuzmiak, A. A. Maradudin, and F. Pincemin, Phys. Rev. B 50, 16835 (1994).

[13] T. Ito and K. Sakoda, Phys. Rev. B 64, 045117 (2001).

[14] E. Moreno, D. Erni, and C. Hafner, Phys. Rev. B 65, 155120 (2002).

[15] C. C. Chang, J. Y. Chi, R. L. Chern, C. C. Chang, C. H. Lin, and C. O. Chang, Phys. Rev. B 70, 075108 (2004).

[16] C. C. Chang, R. L. Chern, C. C. Chang, and R. R. Hwang, Phys. Rev. B 72, 205112 (2005).

[17] C. Kittel, Introduction to Solid State Physics, 7th ed. (John
Wiley \& Sons, New York, 1996).

[18] C. F. Bohren and D. R. Huffman, Absorption and Scattering of Light by Small Particles (John Wiley \& Sons, New York, 1983).

[19] J. W. Demmel, Applied Numerical Linear Algebra (SIAM, Philadelphia, 1997).

[20] J. J. Sakurai, Modern Quantum Mechanics (Addison-Wesley, New York, 1994).

[21] W. L. Barnes, A. Dereux, and T. W. Ebbesen, Nature (London) 424, 824 (2003).

[22] E. Burstein, Polaritons (Pergamon, New York, 1974).

[23] N. Peyghambarian, S. W. Koch, and A. Mysyrowicz, Introduction to Semiconductor Optics (Prentice-Hall, Englewood Cliffs, NJ, 1993).

[24] G. Shvets, Phys. Rev. B 67, 035109 (2003).

[25] H. Raether, Surface Plasmons on Smooth and Rough Surfaces and on Gratings (Springer-Verlag, Berlin, 1988).

[26] W. C. Tan, T. W. Preist, and R. J. Sambles, Phys. Rev. B 62 , 11134 (2000).

[27] W. C. Liu and Ding Ping Tsai, Phys. Rev. B 65, 155423 (2002).

[28] W. A. Murray, S. Astilean, and W. L. Barnes, Phys. Rev. B 69, 165407 (2004).

[29] A. R. McGurn and A. A. Maradudin, Phys. Rev. B 48, 17576 (1993).

[30] W. Zhang, X. L. An Hu, N. Xu, and N. Ming, Phys. Rev. B 54, 10280 (1996). 
[31] E. I. Smirnova, C. Chen, M. A. Shapiro, J. R. Sirigiri, and R. J. Temkin, J. Appl. Phys. 91, 960 (2002).

[32] J. D. Jackson, Classical Electrodynamics, 3rd ed. (John Wiley \& Sons, New York, 1999).

[33] C. A. Balanis, Advanced Engineering Electromechanics (John Wiley \& Sons, New York, 1989).

[34] T. Suzuki and P. K. L. Yu, Phys. Rev. B 57, 2229 (1998).
[35] D. J. Griffiths, Introduction to Electrodynamics, 3rd ed. (Prentice Hall, Englewood Cliffs, NJ, 1999).

[36] J. B. Pendry, L. Martin-Moreno, and F. J. Garcia-Vidal, Science 305, 847 (2004).

[37] P. B. Johnson and R. W. Christy, Phys. Rev. B 6, 4370 (1972).

[38] A. Pinchuk, U. Kreibig, and A. Hilger, Surf. Sci. 557, 269 (2004). 